\title{
The changes in the education of psychiatric nurses: do psychiatrists have a role?
}

\author{
Michael S. JoRSH, Lecturer/Senior Registrar in Psychiatry, University of Keele and \\ City General Hospital, Stoke-on-Trent ST4 7QB
}

The new syllabus for both general and psychiatric nurses, Project 2000, is a manifestation of the gradual revolution taking place in nursing. It attempts to address some long-standing issues which have concerned the profession in several countries. The resolution of these issues will determine the role of nurses as members of the multidisciplinary team and effect cooperation and interactions therein.

\section{Background}

Project 2000 is intended to improve the academic standards of the nursing profession which sees itself as becoming more active in a primary preventive role. The approach to nursing education in the past has been fairly dogmatic and new syllabi must be scrutinised carefully to ensure that the nurse is equipped to work within the clinical environment and the therapeutic team.

Most psychiatrists would share Altshul's (1984) view that an eclectic approach which promotes awareness of all aspects of patients' problems, including the medical and biochemical, is essential. She feels that nursing theories should relate closely to those used by the medical profession. The close ties which psychiatric nurses have always had with the medical profession have only recently been loosened. Nurse educators remain sensitive to this and some show hostility towards psychiatry which can only be counterproductive.

Nursing models or theoretical frameworks are seen by many educators as vehicles towards professionalisation. These models will guide future nursing philosophy. Botha (1989) warns against dogmatism. The differing models reflect the diverse approaches, but some have been adopted with inadequate testing. While they provide a useful framework, further research is needed into their effectiveness.

Hardy (1986) views nursing models as potentially restrictive and suggests that they could promote specialised concepts and a new jargon with deleterious effects on communication between the nurses and both patients and other professions. Models aim to focus on the consumer, but the approach is dictatorial and focuses only on the nursing perspective. She feels that they can be useful aids to discussion and the organisation of thought and that using multiple models could stimulate creative thinking but that they should be modified according to research findings.

\section{The new teaching policies in practice}

Project 2000 has entered its second year. The Student Handbook for Mental Health Nursing of the North Staffordshire College of Nursing and Midwifery (1989) outlines its syllabus which is presumably similar to others. The principles and philosophy of psychiatric nursing defined within it are encouraging. The approach is to view the patients within their social contexts and to use both interactive and therapeutic skills. The view is that the nurse is a practitioner and, by implication, a professional.

Some disturbing aspects of the syllabus require comment. As psychiatry and psychiatric nursing have a commensal relationship, each must be aware of what the practitioners of the future are to be taught and how this could effect their working relationship. We need to be aware of our abilities and limitations to co-operate within the multidisciplinary team.

The syllabus appears to use Johnson's nursing model, based on Systems Theory, as outlined by Botha (1989). Environment and health are discussed in terms of the individual's interactions within the behavioural system to which they belong. Health is seen as a moving state of equilibrium and the system acts in an effort to maintain balance. The major cause of instability within the system is determined and the source of the problem is identified leading to appropriate nursing intervention. Systems theory, while useful, denies the role of the individual in the process of development and treatment of psychiatric conditions.

The Handbook regards the patient (client) as being in a state of "disequilibrium" and interventions are directed towards restoration of equilibrium. The disequilibrium (symptom) does not appear to be linked to a diagnosis. For example, suicidal urges and sleep disturbance may not be linked to depression. Psychiatric syndromes and conventional psychopathology seem to be disregarded. Each individual's problems are dealt with symptomatically. 
Because the syllabus is fairly dogmatic while implying breadth, the student may come to regard theory as truth. Its approach to descriptive psychopathology and psychiatric syndromes is tantamount to treating all people with chest pain in the same way, despite the fact that one may have cardiac problems while another may have local trauma. This dangerous approach may be symptomatic of the need for nurses to distance themselves from medicine in an attempt to confirm their independence.

Cox (1991) describes the medical model which bases management and treatment on the eliciting of signs and symptoms by the clinician (in the case of psychiatric illness, the psychopathology). He regards this approach as valuable even for conditions which do not have a biological cause, but emphasises that psychodynamic and social issues must be considered. He advocates an eclectic approach, using all available models, as psychiatry is based on both the social and biological sciences. Paradoxically, if the medical approach is ignored in order to promote holistic understanding, an important aspect of the individual may be overlooked.

The pioneering work of the North Staffordshire College of Nursing is viewed with well-earned respect nationally. Contact made by other colleges could lead to them adopting the syllabus with very little change. A local issue could therefore become a national one.

\section{Comment}

Nursing is in the midst of a revolution and is moving away from a role subservient to medicine. Moving too far from its roots could render it isolated. Positive links with the past and other professions could be encouraged and their expertise utilised to improve education without hampering the move towards independence. Future professionals will have to work within the multidisciplinary team and must be able to communicate with their colleagues using and understanding a common language. Rigid adherence to a particular theory may prove a hindrance in the clinical setting as theory may not translate well when confronted by clinical reality and the need for a multi-model understanding of patients.

The essence of treatment in the mental health setting is communication - not only between patient and professional, but between the professions. A common language and approach will not detract from their different skills, but will aid communication. Professions sharing knowledge across bound- aries will teach this approach from an individual's earliest exposure to their profession. With psychiatric nurses, this would involve psychiatrists being more closely involved with the teaching of students, not in an attempt to indoctrinate them or to turn them into 'mini-psychiatrists', but to foster communication and to share expertise. Psychiatric nurses could train future psychiatrists in the uses and practices of counselling and creative therapy as well as the use of the problem orientated approach to patient care.

\section{Conclusions}

The evolution of nursing into a profession has led to the nurse becoming an independent practitioner contributing further expertise to the multidisciplinary team. It should not, however, be at the expense of unity within the team. Nursing colleges should make use of the expertise which exists within psychiatry in order to encourage the creation of a common language and improve communication without restricting their unique nursing skills.

All mental health practitioners must be familiar with descriptive psychopathology, being the basis of psychiatric diagnosis, and psychiatric syndromes. Once this knowledge is acquired, individuals are in a position to formulate their own opinions as to its importance. Dogmatism could hamper students' abilities to make informed choices.

\section{Acknowledgement}

With thanks to Professor John Cox for his criticism and advice.

\section{References}

AltShUL, A. (1984) Does good practice need good principles? Nursing Times, 80, 36-38.

BoTHA, M. E. (1989) Theory development in perspective: the role of conceptual frameworks and models in theory development. Journal of Advanced Nursing, 14, 49-55.

Cox, J. L. (1991) Childbirth, Mental Illness and the Medical Model. Proceedings of the Royal College of Physicians of Edinburgh, April 1991 (in print).

HARDY, L. K. (1986) Janforum: Identifying the place of theoretical frameworks in an evolving discipline. Journal of Advanced Nursing, 11, 103-107.

North Staffordshire College of Nursing and MidWIFERY (1989) Mental Health Nursing - Student Handbook. 